\title{
A Rare Case of Heterotopic Ossification of the Patella Tendon in an Adolescent Presenting with Tendon Rupture
}

\author{
Sam Supreeth ${ }^{1} \quad$ Aliya Al-Barwani ${ }^{2} \quad$ Ismail Al Habsi ${ }^{1} \quad$ Suwailim Al Ghanami ${ }^{1}$ Zahra Al Abri ${ }^{3}$ \\ Khadija Al-Adawi ${ }^{2}$ \\ ${ }^{1}$ Department of Orthopaedics, Khoula Hospital, Muscat, Oman \\ 2 Musculoskeletal Radiology, Khoula Hospital, Muscat, Oman \\ 3 Department of Pathology, Khoula Hospital, Muscat, Oman

\begin{abstract}
Address for correspondence Sam Supreeth, MBBS, MS, Department of Orthopaedics, Khoula Hospital, Al Fahal St, Muscat, Oman (e-mail: supreethsam13@gmail.com).
\end{abstract}

Joints 2019;7:131-134.

\begin{abstract}
Keywords

- heterotopic ossification

- adolescent

- patellar tendon

- rupture

Heterotopic ossification is the abnormal formation of mature, lamellar bone in nonosseous tissue such as tendons, ligaments, muscles, and soft tissue. We discuss a rare case of a young adolescent with patellar tendon rupture postheterotopic ossification. A 13-year-old male presented to us with knee pain and inability to extend for 6 weeks following trivial trauma. Preliminary radiological investigations revealed a high riding patella with ossification in the patella tendon. The magnetic resonance scan confirmed the same with patellar tendon disruption and heterogeneous ossification. He underwent surgery with patella tendon repair, augmentation with autograft, and had complete recovery at 6 months' follow-up.
\end{abstract}

\section{Introduction}

Heterotopic ossification (HO) is a diverse pathological process resulting in the abnormal formation of mature, lamellar bone in any form of nonosseous tissue such as the muscle, tendon, ligament, vascular tissue, soft tissues, and even brain. ${ }^{1}$ The term "heterotopic ossification" is derived from Greek (heteros and topos) and Latin (ossification) words meaning "bone formation in other locations." 2

The occurrence of $\mathrm{HO}$ in the knee is infrequent; the most common sites of appearance, in decreasing order, are the hips, knees, shoulders, and elbows. ${ }^{3} \mathrm{HO}$ is known to occur around the knee postarthroplasty, intramedullary nailing, anterior cruciate ligament reconstruction, and knee injury without fracture. $^{4}$

We report a case of patellar tendon rupture in a patient, which was repaired and augmented with a semitendinosus autograft. To the best of our knowledge, this is the first report of patellar tendon rupture in a young adolescent post-HO.

received

January 20, 2020

accepted after revision

April 13, 2020

published online

May 20, 2020

\section{Case Presentation}

A 13-year-old male referred to us with pain and an inability to extend the right knee. He had a trivial history of fall 6 weeks prior, following which he heard a "pop" sound from his knee. The trauma resulted in swelling associated with pain. He gave a history of repetitive injury to the same knee due to sporting activities in the recent past.

Clinically, the patient held the knee in flexion with mild effusion of the joint. The patella was high-riding compared with the normal side. The extension mechanism of the knee had failed with an active range of movement between 60 and 110 degrees. A palpable discontinuity was noted in the patella tendon with irregular hard swellings.

An initial radiograph ( - Fig. 1) in anteroposterior and lateral view confirmed the patella alta with irregular, well-defined radiodense opacities at the site of the disrupted patella tendon with zoning appearance; the periphery was denser than the center. Further evaluation with ultrasound confirmed a patella tendon rupture and a gap was seen (-Fig. 2). Magnetic

(c) 2020. The Author(s).

This is an open access article published by Thieme under the terms of the Creative Commons Attribution-NonDerivative-NonCommercial-License, permitting copying and reproduction so long as the original work is given appropriate credit. Contents may not be used for commercial purposes, or adapted, remixed, transformed or built upon. (https://creativecommons.org/ licenses/by-nc-nd/4.0/)

Georg Thieme Verlag KG, Rüdigerstraße 14, 70469 Stuttgart, Germany 


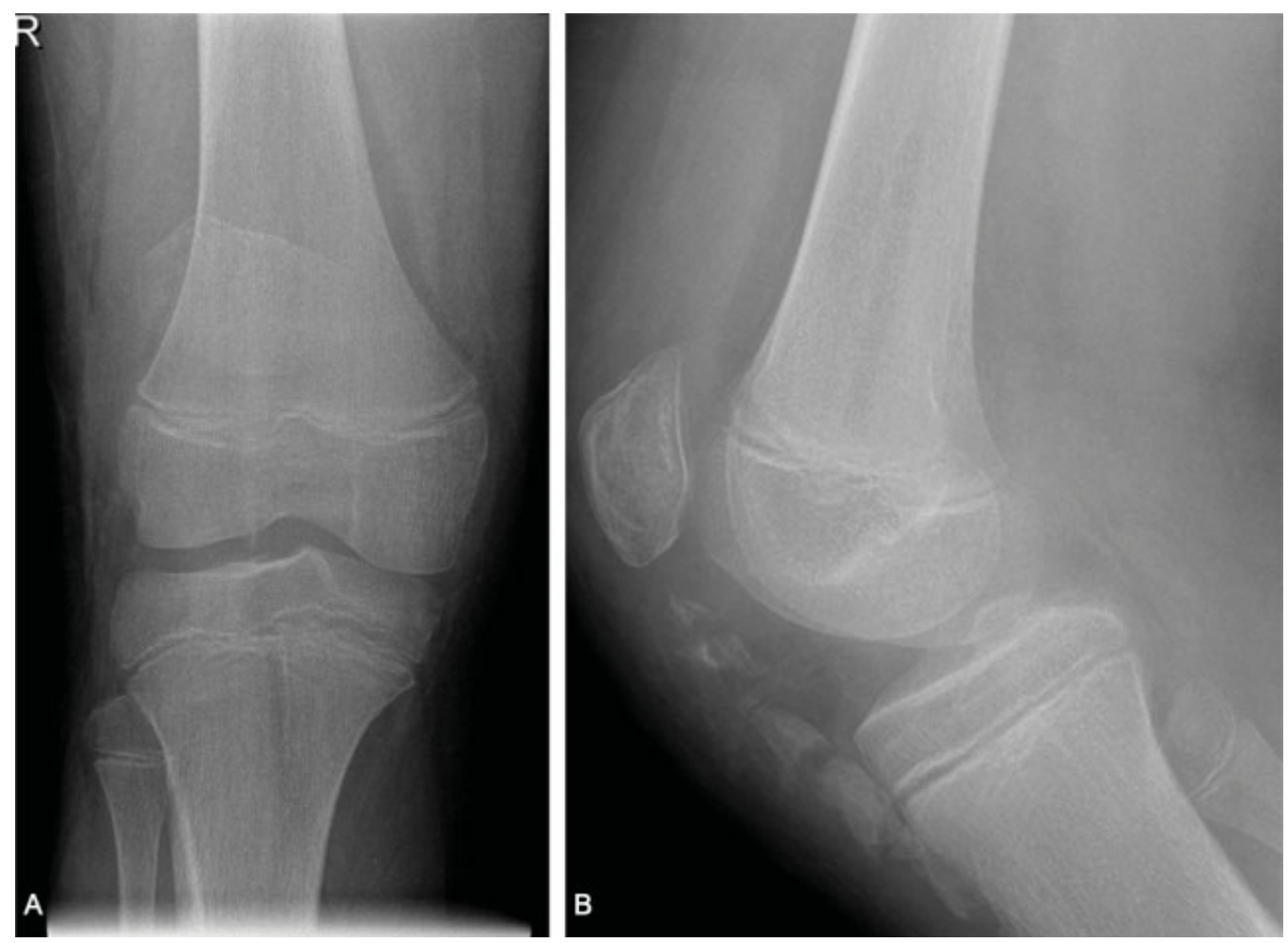

Fig. 1 (A) Anteroposterior radiograph of the right knee showing a high-riding patella in-keeping with patella alta. No evidence of fractures. (B) Lateral radiograph of the right knee showing coarse chunky radiodensities at the site of the patella tendon. Some of the superior radiodensities are showing "zoning phenomena" where the periphery is denser, and the center more lucent.

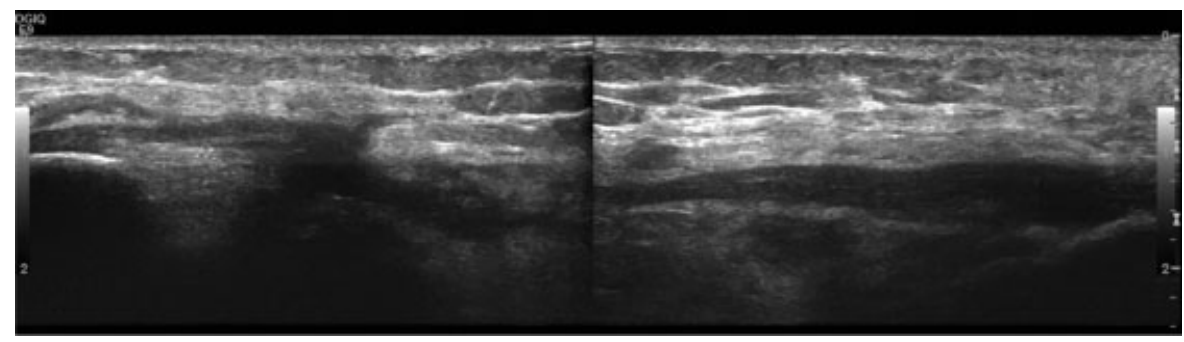

Fig. 2 Ultrasound grayscale imaging showing disruption of the patella tendon with a visualized, ill-defined hypoechoic collection at the gap.

resonance imaging (MRI) showed the radiodense opacities to be of low signal intensity peripherally T1- and T2-weighted imaging with central intensity matching that of the tibia and femoral bone (-Fig. 3). The hematological and biochemical laboratory results including serum calcium were within normal parameters.

Surgically, the patellar tendon edges were approximated, sutured, and further augmented with semitendinosus autograft of the same side. The semitendinosus was looped across the inferior pole of the patella and the tibial tuberosity in a figure of "8." Intraoperatively, two large pieces of calcified tissue measuring $45 \times 20 \times 10$ and $30 \times 15 \times 7 \mathrm{~mm}$, were found in the patellar tendon. Histopathological evaluation revealed dense fibrocollagenous tissue with ossific foci, with lamellar bone, enclosing fatty marrow and woven bone with chondroid elements, typical of HO (-Fig. 4).

The patient was discharged with a slab above the knee at 20 degrees to prevent flexion and was on regular follow-up. The slab was discontinued at 2 weeks postoperatively, con- tinuous passive motion allowed, and the patient started full weight bearing by 6 weeks. At the latest review 6 months postoperatively, the patient showed a full active range of movement with no extension lag.

\section{Discussion}

$\mathrm{HO}$ is mainly divided into acquired and genetic of which the former is more commonly related to trauma. ${ }^{3}$ Up to $75 \%$ of the cases have a history of significant trauma, whereas in our case the etiology was repetitive microtrauma. ${ }^{5}$

Men are more commonly affected by a ratio of 3:2. Classically, nongenetic $\mathrm{HO}$ is seen in young adults in the second and third decade of life. Our case is the youngest in reviewing the literature. $^{5}$

Clinically, most nongenetic cases present with localized pain, swelling, and tenderness in the initial stage, followed by firm swelling and restricted adjacent joint movement. ${ }^{5}$ There has been no report of cases with patellar tendon rupture. 

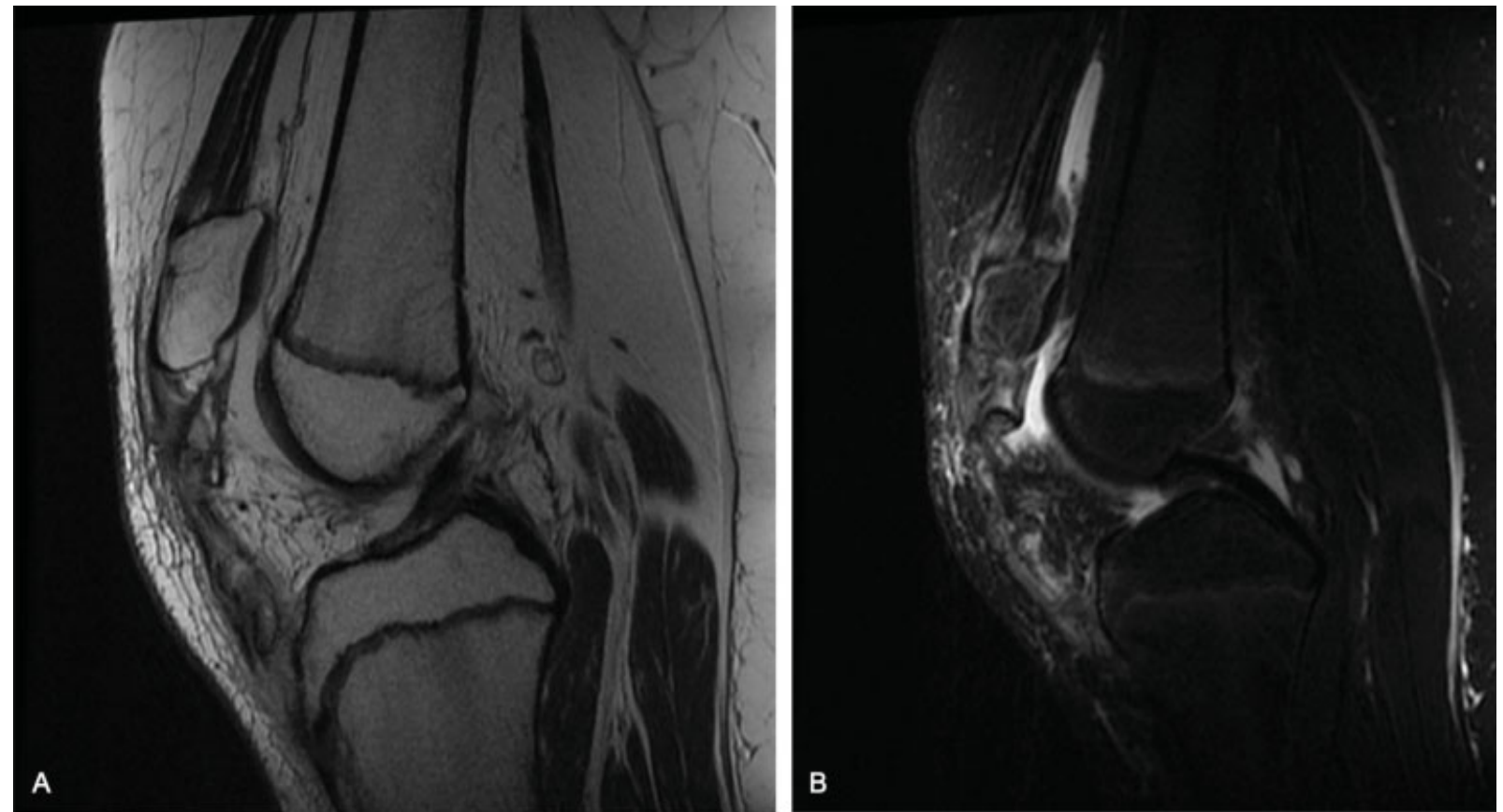

Fig. 3 (A) Sagittal oblique T2-weighted imaging of the right knee showing irregular patella tendon with high signal intensity. Images demonstrate a patella alta, ossifications seen within the irregular patella tendon which are showing peripheral hypointensity with a central area that is more hyperintense, in-keeping with zoning phenomena. These ossifications are matching the signal intensity of the native bones with cortical hypointensity and central hyperintensity. (B) Sagittal selected magnetic resonance imaging (MRI) short tau inversion recovery (STIR)weighted imaging demonstrating an irregular disrupted patella with internal high-signal intensity edema, joint effusion, and hypointense ossifications, more hypointense peripherally.
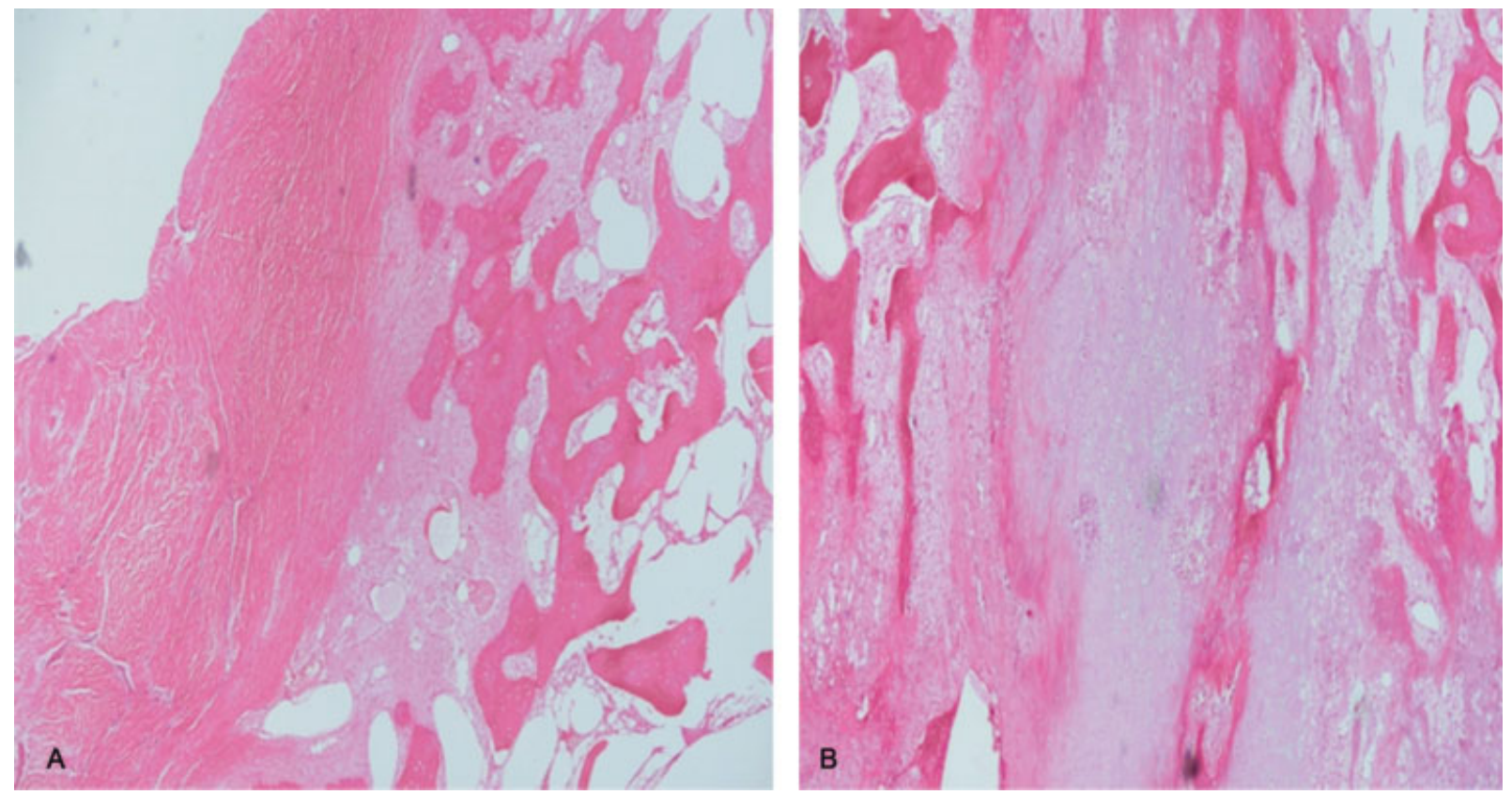

Fig. 4 Histology image showing (A) calcification in the tendon and (B) lamellar ossification with fatty marrow and chondroid elements (hematoxylin and eosin [H-E] staining. Magnification 20x).

The radiograph revealed discontinuity of patella tendon with well-defined opacities classically described as "eggshell" calcifications with regards to HO. ${ }^{5}$ A minimum of 6 weeks is required for detecting calcification after the inciting trauma, hence furthering the hypothesis of repetitive microtrauma over a prolonged period than mentioned. ${ }^{3}$
The appearance on MRI as described above in our case, with no evidence of underlying mass lesion, soft tissue enhancing component, and underlying aggressive native bone erosion, all favor a benign process. HO in early intermediate stages may show postcontrast central enhancement within the calcifications. This is likely due to the central active part of 
134 Heterotopic Ossification of the Patella Tendon in an Adolescent Presenting with Tendon Rupture Supreeth et al.

bone formation whereas the periphery is mature lamellated cortical bone, that is, zoning phenomena. ${ }^{5,6}$

Histology revealed characteristic bone maturation and spatial zonation with mature lamellar bone at the periphery in the continuum of woven bone. ${ }^{7}$ No definite etiopathogenesis exists for $\mathrm{HO}$, the hypothesis of vascular insufficiency resulting from repetitive trauma leading to collagen deposition and calcium deposition making the patellar tendon susceptible to rupture. ${ }^{8}$ Specific tissues in the body are more vulnerable to HO (brain, hip, elbow, spinal cord); however, it is not understood why specific tissues are commonly susceptible to these lesions whereas others not. Our described case is an infrequent manifestation/location of a widely described entity (HO). ${ }^{5}$

Treatment, in this case, involved surgical excision, repair, and augmentation with autograft and prophylactically nonsteroidal anti-inflammatory drugs used to prevent recurrence. ${ }^{5,9}$

\section{Conclusion}

$\mathrm{HO}$ in tendinous sites usually occurs at the enthesis, the point of bone insertion. It classically manifests as coarse, chunky ossification with zoning phenomena. It happens postacute trauma or postrepetitive chronic injury weakening the underlying tendon predisposing it to tendinopathies including complete and partial tears.
Conflict of Interest

None declared.

\section{References}

1 Balboni TA, Gobezie R, Mamon HJ. Heterotopic ossification: pathophysiology, clinical features, and the role of radiotherapy for prophylaxis. Int J Radiat Oncol Biol Phys 2006;65(05):1289-1299

2 Al-Zahrawi. Albucasis on Surgery and Instruments. Berkeley: University of California Press; 1972

3 Mujtaba B, Taher A, Fiala MJ, et al. Heterotopic ossification: radiological and pathological review. Radiol Oncol 2019;53(03): 275-284

4 Cakici H, Hapa O, Ozturan K, Guven M, Yucel I. Patellar tendon ossification after partial patellectomy: a case report. J Med Case Reports 2010;4:47

5 Meyers C, Lisiecki J, Miller S, et al. Heterotopic ossification: a comprehensive review. JBMR Plus 2019;3(04):e10172

6 Zagarella A, Impellizzeri E, Maiolino R, Attolini R, Castoldi MC. Pelvic heterotopic ossification: when CT comes to the aid of MR imaging. Insights Imaging 2013;4(05):595-603

7 Cocks M, Mohan A, Meyers CA, et al. Vascular patterning in human heterotopic ossification. Hum Pathol 2017;63:165-170

8 Vaishya R, Maduka CO, Agarwal AK, Vijay V, Vaish A. Heterotopic ossification of tendo achilles: an uncommon clinical entity. J Orthop Case Rep 2019;9(02):45-47

9 Ritter MA, Sieber JM. Prophylactic indomethacin for the prevention of heterotopic bone formation following total hip arthroplasty. Clin Orthop Relat Res 1985;(196):217-225 